\title{
ORIGINAL ARTICLE \\ Risks of undernutrition and malnutrition in hospitalized pediatric patients with spinal cord injury
}

\author{
Y-J Wang ${ }^{1,2}$, H-J Zhou ${ }^{1}$, P-J Liu ${ }^{3}$, G-L Liu ${ }^{1}$, Y Zheng ${ }^{1}$, B Wei ${ }^{1}$, C-X Hao ${ }^{1}$, Y Zhang ${ }^{1}$, H-Q Kang ${ }^{1}$, X-L Lu ${ }^{1}$ \\ and Y Yuan ${ }^{1}$
}

Study design: Prospective study.

Objectives: To describe the nutritional risk/status of Chinese children with spinal cord injury (SCI) at admission and determine the relationship between nutritional risk/status and demography/SCl characteristics.

Setting: China Rehabilitation Research Center, Beijing, China.

Methods: Baseline clinical data, appetite level, anthropometric measurements and Screening Tool for the Assessment of Malnutrition in Pediatrics (STAMP) scores were obtained for pediatric SCI patients. The relationships among the demographic/SCl characteristics and STAMP score and $z$-scores of weight-for-age (WAZ), height-for-age (HAZ) and body mass index-for-age (BAZ) were assessed. The risk of undernutrition was compared with actual nutritional status.

Results: Forty-five children including 12 boys and 33 girls were included. The risks of undernutrition using the STAMP tool and malnutrition were $51.1 \%$ and $55.6 \%$, respectively. Children with different demographic characteristics had similar nutritional status and risk of malnutrition. The risk of undernutrition was associated with nutritional status, including WAZ $(P<0.001), \operatorname{HAZ}(P=0.001)$, BAZ $(P<0.001)$ and appetite level $(P<0.001)$. Compared with nutritional status, STAMP had a sensitivity of $100 \%$, a specificity of $73.3 \%$ and an overall agreement of $82.2 \%$. As the duration of $\mathrm{SCl}$ increased, the risks of overweight and stunting increased.

Conclusions: Nutritional screening in all pediatric SCl patients should be performed periodically. The decreasing trends in nutritional status and appetite level after $\mathrm{SCl}$ require special attention. The STAMP may be an alternative method for assessing nutritional status in Chinese children with $\mathrm{SCl}$.

Spinal Cord (2017) 55, 247-254; doi:10.1038/sc.2016.113; published online 9 August 2016

\section{INTRODUCTION}

Generic national and international guidelines ${ }^{1}$ have highlighted the importance of nutritional screening as the first step in fighting childhood malnutrition, but only a small proportion of at-risk children with spinal cord injury (SCI) are referred for nutritional assessment and interventions. ${ }^{1}$ Current awareness among the physicians and nurses regarding the importance of screening and treating malnutrition in children with a SCI appears to be low. ${ }^{2}$ In addition, malnourished SCI patients may present with a variety of atypical symptoms, with the result being that malnutrition remains undetected. ${ }^{3}$ The literature on the assessment of nutritional status in children with SCI is limited. Studies have found that almost half of children with SCI $(45.5 \%)$ are at risk of malnutrition, and the prevalence of overnutrition is high (overweight, 41.1\%; obese, 25.5\%). ${ }^{4,5}$ However, the relationships between nutritional risk and pediatric characteristics after SCI such as age, gender, years since SCI, level of injury, completeness of injury, American Spinal Injury Association Impairment Scale (AIS) and walking ability are unclear.

The Screening Tool for the Assessment of Malnutrition in Pediatrics (STAMP) is the first approved screening tool to identify risk of malnutrition in children in Great Britain ${ }^{4,6}$ and has also been validated in the Children's Hospital in China.,8 STAMP is intended for use by the nursing staff as a first-line screen, and the validity and reliability of this tool has been confirmed in SCI children. ${ }^{5}$ The STAMP incorporates three components, all of which are recognized indices or symptoms of undernutrition: the presence of a clinical diagnosis that has nutritional implications, estimated recent nutritional intake, and differences in weight/height centile chart. ${ }^{5}$ In the first step, the diagnosis table on the STAMP website is used to determine whether the child's condition has any nutritional implications, and diseases are categorized into three groups according to the influence on the nutritional status of the child (without nutritional consequence -0 points, potential nutritional consequences-2 points, some nutritional consequences- 3 points). The second step of the evaluation is to assess the child's current nutritional intake. Nutritional intake is assessed as none (3 points), recently decreased/poor ( 2 points) or no change/good ( 0 points). In the final step, the centile reference tables from children without SCI are used to determine how many columns/centiles apart the height and weight are: $>3$ centile spaces $/ \geqslant 3$ columns apart ( 3 points), $>2$ centile spaces/ $=2$ columns apart ( 1 point) and $0-1$ centile space/column apart ( 0 point). The scores from steps 1 to 3 are summed to calculate the child's overall risk of malnutrition: high risk ( $\geqslant 4$ points), medium risk (2-3 points), and low risk (0-1 point) (Figure 1).

${ }^{1}$ Department of Spinal Cord Injury Rehabilitation, China Rehabilitation Research Center, Beijing, China; ${ }^{2}$ School of Rehabilitation Medicine, Capital Medical University, Beijing, China and ${ }^{3}$ Department of Clinical Nutrition, Peking Union Medical College Hospital, China Academic Medical Science and Peking Union Medical College, Beijing, China Correspondence: Dr H-J Zhou, Department of Spinal Cord Injury Rehabilitation, China Rehabilitation Research Center, No. 10, Jiao Men Bei Lu, Feng Tai District, Beijing 100068, China.

E-mail: crrczhouhongjun@126.com

Received 18 December 2015; revised 11 May 2016; accepted 28 May 2016; published online 9 August 2016 
The present study was conducted to expand our knowledge of the nutritional risk/status of children with SCI and determine the relationship between nutritional risk/status and demography/SCI characteristics in pediatric inpatients ${ }^{9,10}$ in order to generate the much-needed data on strategies for improving the nutritional status of children with SCI. We also aimed to evaluate the concurrent validity of a

\begin{tabular}{|l|l|}
\hline \multicolumn{2}{|c|}{ Step 1- Diagnosis } \\
\hline $\begin{array}{l}\text { Does the child have } \\
\text { a diagnosis that } \\
\text { has any nutritional } \\
\text { implications? }\end{array}$ & Score \\
\hline Definitely & 3 \\
\hline Possibly & 2 \\
\hline No & 0 \\
\hline
\end{tabular}

\section{Step 2 - Nutritional intake}

What is the child's nutritional intake?

Score

\begin{tabular}{|l|l|}
\hline None & 3 \\
\hline $\begin{array}{l}\text { Recently } \\
\text { decreased/poor }\end{array}$ & 2 \\
\hline No change/good & 0 \\
\hline
\end{tabular}

\section{Step 3 - Weight and height}

Use a growth chart or the centile quick reference tables to Score determine the child's measurements

$>3$ centile spaces/ $\geq 3$ columns apart (or 3 weight $<2^{\text {nd }}$ centile)

$>2$ centile spaces/ $=2$ columns apart 0 to 1 centile spaces/ columns apart

\section{Step 4 - Overall risk of malnutrition}

Add the scores from steps 1-3 together to calculate the overall risk of malnutrition

Score

High risk

$\geq 4$

Medium risk

$2-3$

Low risk

$0-1$

\section{Step 5 - Care plan}

Develop a care plan based on the child's overall risk of malnutrition

High risk

n Take action

= Refer to a Dietitian, nutritional support team or consultant

= Monitor as per care plan
Medium risk

= Monitor nutritional intake for 3 days

= Repeat STAMP screening after 3 days

n Amend care plan as required
Low risk

= Continue routine clinical care

= Repeat STAMP screening weekly while child is an in-patient

= Amend care plan as required

b

\begin{tabular}{|c|c|c|}
\hline Definite nutritional implications & $\begin{array}{l}\text { Possible nutritional } \\
\text { implications }\end{array}$ & $\begin{array}{l}\text { No nutritional } \\
\text { implications }\end{array}$ \\
\hline $\begin{array}{l}\text { " Bowel failure, intractable diarrhoea } \\
\text { " Burns and major trauma } \\
\text { " Crohn's disease } \\
\text { = Cystic fibrosis } \\
\text { " Dysphagia } \\
\text { = Liver disease } \\
\text { " Major surgery } \\
\text { " Multiple food allergies/intolerances } \\
\text { = Oncology on active treatment } \\
\text { " Renal disease/failure } \\
\text { " Inborn errors of metabolism }\end{array}$ & $\begin{array}{l}\text { = Behavioural eating problems } \\
\text { " Cardiology } \\
\text { " Cerebral palsy } \\
\text { " Cleft lip and palate } \\
\text { " Coeliac disease } \\
\text { = Diabetes } \\
\text { " Gastro-oesophageal reflux } \\
\text { = Minor surgery } \\
\text { " Neuromuscular conditions } \\
\text { " Psychiatric disorders } \\
\text { " Respiratory syncytial virus (RSV) } \\
\text { " Single food allergy/intolerance }\end{array}$ & $\begin{array}{l}\text { " Day case surgery } \\
\text { " Investigations }\end{array}$ \\
\hline
\end{tabular}

Figure 1 Screening Tool for Assessment of Malnutrition in Pediatrics (STAMP). A full color version of this figure is available at the Spinal cord journal online. 
STAMP (a tool for assessing risk of malnutrition) in the assessment of nutritional status in pediatric SCI patients because of good association between STAMP and $z$-scores of weight-for-age (WAZ), height-forage (HAZ) and body mass index-for-age (BAZ), which are the indicators of nutrition status.

\section{MATERIALS AND METHODS}

Patient recruitment

Children admitted for rehabilitation treatment between July 2014 and July 2015 to the China Rehabilitation Research Center (CRRC) were enrolled in the present study. Nine children were able to walk, and the others were wheelchair users. The CRRC is a state-owned institution affiliated with the China Disabled Persons' Federation that provides comprehensive rehabilitation and social services to the disabled. The study inclusion criteria were children aged between 1 and 12 years of age, presence of SCI and inpatients. We divided the children into four groups (boys $0-5$ years/6-12 years and girls $0-5$ years/6-12 years). Time since injury was divided into acute injuries $(<1$ year) and chronic injuries (1-5 years). Patients were excluded if they had fever or diarrhea on admission, a history of hepatopathy, nephropathy or formal dietetic assessment and nutritional therapy that may have introduced a bias toward nutrition status from medical records.

\section{Data collection}

Baseline demographics (age and gender) and clinical characteristics, including weight, height, appetite level, causes of SCI, duration of SCI, level of SCI, AIS score and walking ability, were recorded. Weight and height were measured and recorded for all inpatient children within the first $48 \mathrm{~h}$ of admission. Weight was measured in the morning before breakfast with light clothing and after voiding and was determined to the nearest $0.1 \mathrm{~kg}$ by a calibrated digital scale. The length was recorded to the nearest $0.1 \mathrm{~cm}$ from the bottom of the right heel to the crown of the head, lying down on a firm surface with their head and foot against a hard board. ${ }^{11,12}$ If the child was $\geqslant 2$ years old, their length was measured and $0.7 \mathrm{~cm}$ was subtracted to convert it to height. ${ }^{5}$ The appetite level ${ }^{4}$ (percentage of meals eaten) was assessed by discussion with the parents or caregivers. We used the question as an item of the nutritional risk screening tool, STRONGkids (Screening Tool for Risk on Nutritional Status and Growth), which included 'reduced food intake during the past few days (3 days) before admission (not including fasting for an elective surgical procedure)'.9 The food intake included type, volume and frequency of liquid and solid feedings for infants and frequency of eating and a brief description of a typical day's intake for children. ${ }^{13}$ Then the parents or caregivers were asked to estimate a percentage of recent average meals eaten (none $=0 \%$, half $=50 \%$, $\operatorname{good}=100 \%$ ).

\section{Definition of malnutrition risk}

The STAMP scores were calculated by the same physician, and the risk of undernutrition was determined from the following published criteria: STAMP score of $0-1$ point indicates low risk, $2-3$ points indicates moderate risk, and more than 4 points indicates a high risk of malnutrition. ${ }^{14}$

\section{Definition of nutritional status}

In children, nutritional status was determined upon admission using the WAZ, HAZ and BAZ scores, which were calculated using the software WHO AnthroPlus version 1.0.4 (World Health Organisation, Geneva, Switzerland) for children. ${ }^{15}$ The WHO AnthroPlus was developed to facilitate the application of the WHO Reference 2007 for 5-19-year-old children to monitor the growth, which showed continuity with the WHO Child Growth Standards for $0-5$ years (www.who.int/childgrowth). These are included in AnthroPlus for the three indicators, that is, weight-for-age, height-for-age and BMI-for-age. This software enables monitoring of the growth of individuals and populations of children from birth to 19 years of age. However, the WHO 2007 reference does not provide weight reference values for children aged $>10$ years. Therefore,

Table 1 Comparison of nutritional indices in children of different age groups

\begin{tabular}{|c|c|c|c|c|c|}
\hline \multirow[t]{3}{*}{ No. of subjects } & \multicolumn{4}{|c|}{ Groups } & \multirow{3}{*}{$\frac{\text { Significance }}{\text { P-value }}$} \\
\hline & Boys (0-5 years) & Girls (6-12 years) & Boys (6-12 years) & Girls ( $0-5$ years) & \\
\hline & $(N=7)$ & $(N=9)$ & $(N=5)$ & $(\mathrm{N}=24)$ & \\
\hline Weight-for-age (z-score) $)^{a}$ & $0.79 \pm 1.22$ & $-0.27 \pm 1.00$ & $0.46 \pm 0.66$ & $0.20 \pm 1.33$ & 0.368 \\
\hline Height-for-age ( $z$-score $)^{a}$ & $0.58 \pm 1.10$ & $-0.01 \pm 0.73$ & $0.70 \pm 0.56$ & $0.34 \pm 1.25$ & 0.618 \\
\hline BMI-for-age ( $z$-score $)^{\mathrm{a}}$ & $0.61 \pm 1.38$ & $-0.44 \pm 1.45$ & $0.23 \pm 1.25$ & $-0.02 \pm 1.30$ & 0.468 \\
\hline Scores of STAMPa & $1.14 \pm 1.21$ & $2.22 \pm 1.99$ & $1.60 \pm 1.52$ & $1.50 \pm 1.64$ & 0.597 \\
\hline
\end{tabular}

Abbreviations: BMI, body mass index; STAMP, Screening Tool for the Assessment of Malnutrition in Pediatrics.

avalues are mean \pm s.d.

$P$-values were determined with the use of analysis of variance (weight-for-age, height-for-age and body mass index-for-age) or Kruskal-Wallis nonparametric test (scores of STAMP)

Table 2 Comparison of clinical and nutritional indices with risk of malnutrition ${ }^{\mathrm{a}}(n=45)$

\begin{tabular}{|c|c|c|c|c|c|c|}
\hline \multirow[t]{2}{*}{ Clinical and nutritional indices } & \multicolumn{3}{|c|}{ Risk of malnutrition } & \multicolumn{3}{|c|}{ Significance } \\
\hline & $\begin{array}{c}\text { Low risk } \\
\text { (STAMP score } \leqslant 1)\end{array}$ & $\begin{array}{c}\text { Medium risk } \\
\text { (STAMP score 2-3) }\end{array}$ & $\begin{array}{c}\text { High risk } \\
\text { (STAMP score } \geqslant 4)\end{array}$ & $\mathrm{P}$-value & $r^{c}$ & P-value for association \\
\hline Weight-for-age (z-score) & $0.97 \pm 0.88^{a}$ & $-0.17 \pm 0.92^{b}$ & $-1.58 \pm 0.78^{c}$ & 0.000 & -0.583 & 0.000 \\
\hline Height-for-age (z-score) & $0.90 \pm 0.99^{a}$ & $-0.14 \pm 0.77^{b}$ & $-0.36 \pm 1.29^{b}$ & 0.002 & -0.392 & 0.001 \\
\hline BMI-for-age (z-score) & $0.69 \pm 0.87^{a}$ & $-0.20 \pm 1.27^{b}$ & $-2.08 \pm 0.58^{c}$ & 0.000 & -0.533 & 0.000 \\
\hline Appetite (\% eaten) & $100^{\mathrm{a}}$ & $75.28 \pm 16.04^{b}$ & $72.00 \pm 14.40^{b}$ & 0.000 & -0.773 & 0.000 \\
\hline Motor scores & $58.68 \pm 17.42$ & $58.89 \pm 18.92$ & $53.2 \pm 6.10$ & 0.795 & -0.011 & 0.932 \\
\hline
\end{tabular}

Abbreviations: BMI, body mass index; STAMP, Screening Tool for the Assessment of Malnutrition in Pediatrics.

aValues in the same row with superscript letters a-c are significantly different, $P<0.05$ (Tukey's honest significant difference)

${ }^{b} P$-values were determined with the use of analysis of variance (weight-for-age, height-for-age and body mass index-for-age) or Kruskal-Wallis nonparametric test (appetite and motor scores).

cKendall's $\tau$ correlation coefficient was used for ordinal data. 
$z$-score values for weight-for-age were calculated in order to use it as reference values. ${ }^{16}$

To compare mild/moderate malnutrition appropriately, the nutritional status was classified as follows: mild or moderate undernutrition for -2.00 s.d. $\leqslant$ BAZ $/ \mathrm{WAZ} / \mathrm{HAZ}<-1.00$ s.d. $\quad$ or $\quad-3.00$ s.d. $\leqslant \mathrm{BAZ} / \mathrm{WAZ} / \mathrm{HAZ}<-2$ s.d., respectively; normal when BAZ/WAZ/HAZ values were between -1 s.d. and +1 s.d.; overweight when the $B A Z$ was between +1 s.d. and +2 s.d.; and obese when the BAZ score $>2.00$ s.d. ${ }^{17,18}$ When more than one anthropometric index (WAZ, HAZ and BAZ) was used for classification of nutritional status, the lowest value was used for classification.

Criterion for STAMP applicability. We tested the concurrent validity between the risk of malnutrition determined by STAMP and nutrition status. In order to calculate the diagnostic values (sensitivity, specificity and positive- and negativepredictive values (PPV and NPV, respectively)) of the STAMP, we combined the medium-/high-risk categories and mild/moderate undernutrition in contingency tables.

The study protocol was approved by the Research Ethics Committee of the institutions participating in the study, and at the time of enrollment, the protocol and nature of study were explained in detail to parents or caregivers and written informed consent was obtained.

\section{Statistical analyses}

For baseline and anthropometric data, descriptive statistics were used. The quantitative variables were tested by one sample Kolmogorov-Smirnov for symmetrical or asymmetrical distribution. The risks of undernutrition with nutritional indices, including symmetrical variables (HAZ, BAZ and WAZ and year since SCI), were compared by Student's $t$ test or analysis of variance, and asymmetrical variables (appetite level and motor scores) were compared by Kruskal-Wallis nonparametric test and Tukey's honest significant difference test. The same statistical methods were applied to compare demographic characteristics with nutritional indices. Pearson chi-squared test or Fisher's exact test was used as appropriate to assess associations between risk of undernutrition and demographic/SCI characteristics. Cohen's kappa statistic was used for determining the applicability of STAMP for assessment of actual nutritional status. Statistical significance was set at 5\% $(P<0.05)$. To calculate the diagnostic values of STAMP for nutritional status, its sensitivity, specificity, PPV and NPV were calculated on contingency tables. Statistical analysis was performed using SPSS version 18.0 for Windows (SPSS Inc., Chicago, IL, USA).

\section{RESULTS}

The study population included 12 boys, including those of young age $(7 / 12,58.0 \%)$ and older age $(5 / 12,42.0 \%)$, and 33 girls, including those of young age $(9 / 33,27.3 \%)$ and older age $(24 / 33,72.7 \%)$. The duration of SCI was $<1$ year in 22 patients $(48.9 \%)$ and $1-5$ years in 23 patients $(51.1 \%)$. The prevalences of paraplegia and tetraplegia were $88.9 \%(40 / 45)$ and $11.1 \%(5 / 45)$, respectively. The most frequent AIS type at admission was A $(29 / 45 ; 64.4 \%)$, followed by D (9/45; $20.0 \%)$. Approximately 30 of $45(66.7 \%)$ children had a SCI owing to trauma. None of the children had malnutrition as the main cause of hospital admission.

\section{Relationship between demographic characteristics and nutritional indices}

When we compared the demographic characteristics with nutritional indices, the mean WAZ, HAZ and BAZ values and STAMP scores were not significantly different among the different gender and age groups $(P=0.368$ for WAZ, $P=0.618$ for HAZ, $P=0.468$ for BAZ and $P=0.597$ for STAMP scores; Table 1$)$.

\section{Risk profile for undernutrition based on STAMP scores}

With the use of the STAMP, 22 (48.9\%) children were classified as low risk (STAMP: 0-1), $18(40.0 \%)$ were classified as medium risk (STAMP: 2-3) and 5 (11.1\%) were classified as high risk (STAMP:
4-5). There were no obvious motor score differences among these three groups of children $(P=0.932)$. The mean WAZ, HAZ and BAZ values and appetite levels were significantly different among the three different STAMP groups $(P<0.001, \quad P=0.002, \quad P<0.001$ and

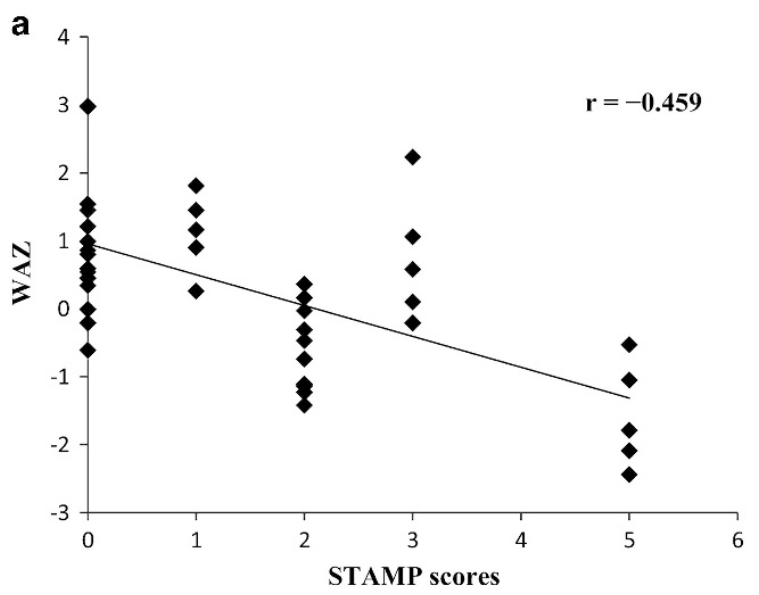

Linear correlation between anthropometric indices(WAZ) and STAMP scores(on admission)

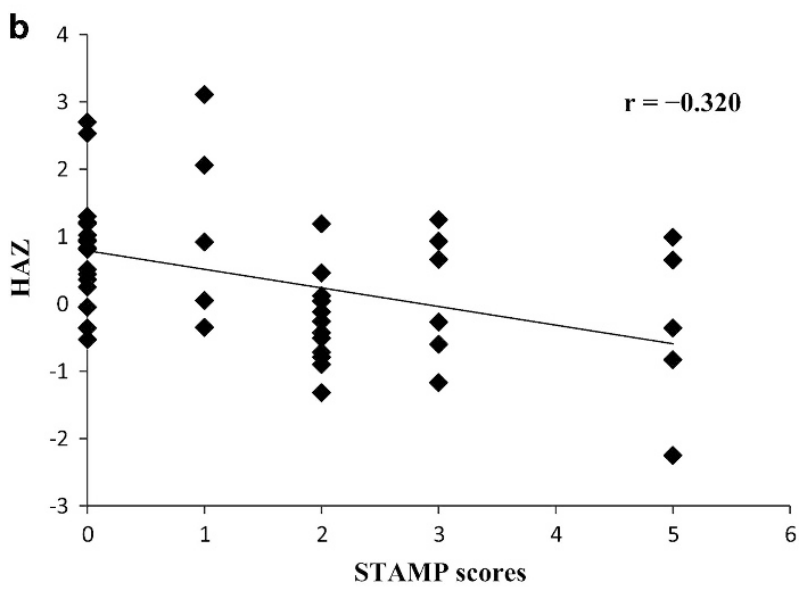

Linear correlation between anthropometric indices(HAZ) and STAMP scores(on admission)

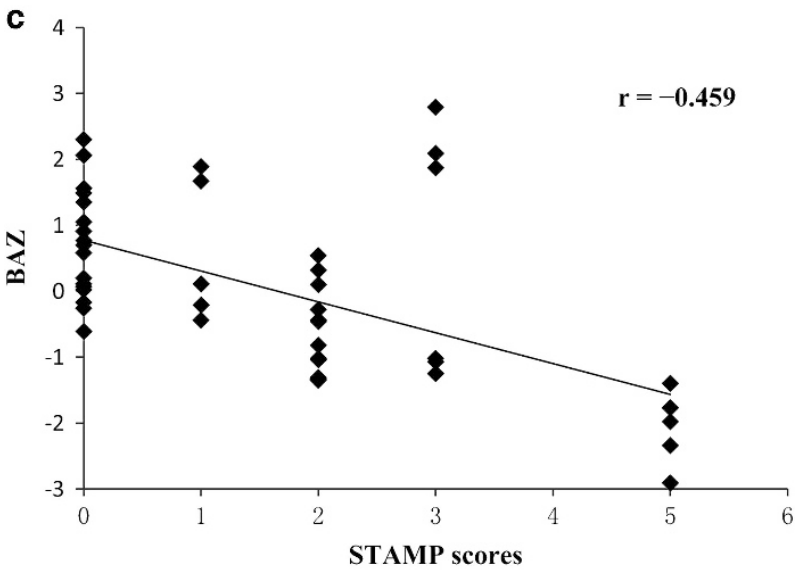

Linear correlation between anthropometric indices(BAZ) and STAMP scores(on admission)

Figure 2 (a) Linear correlation between anthropometric indices (WAZ) and STAMP scores (on admission). (b) Linear correlation between anthropometric indices (HAZ) and STAMP scores (on admission). (c) Linear correlation between anthropometric indices (BAZ) and STAMP scores (on admission). 
$P<0.001$, respectively). These values decreased with increasing degree of malnutrition risk (WAZ: $r=-0.583, P<0.001$, HAZ: $r=-0.392$, $P=0.001$, BAZ: $r=-0.533, P<0.001)$ and appetite $(r=-0.773$, $P<0.001$; Table 2). In addition, STAMP scores also showed a significant negative correlation with WAZ, HAZ and BAZ levels in linear regression analysis $(r=-0.611, P<0.001 ; r=-0.320, P=0.005$; and $r=-0.459, P<0.001$, respectively; Figures $2 \mathrm{a}-\mathrm{c})$.

Relationship between risk of undernutrition and SCI characteristics When we compared the groups of patients according to no risk of undernutrition (STAMP score $\leqslant 1$ ) and at risk for undernutrition (STAMP score $\geqslant 2$ ), there were no significant differences in demographic characteristics (gender and age) or SCI characteristics (cause of injury, years since SCI, level of injury, completeness of injury, AIS

Table 3 Relationship between risk of undernutrition and demography/ $\mathrm{SCl}$ characteristics

\begin{tabular}{|c|c|c|c|}
\hline \multirow{4}{*}{$\begin{array}{l}\text { Demography/SCI } \\
\text { characteristics }\end{array}$} & \multicolumn{2}{|c|}{ Groups $^{\mathrm{a}}$} & $\mathrm{P}$-value \\
\hline & Not at risk & At risk & \\
\hline & (STAMP score $\leqslant 1)$ & $(S T A M P$ score $\geqslant 2)$ & \\
\hline & $\mathrm{n}=22$ & $\mathrm{n}=23$ & \\
\hline
\end{tabular}

Gender $(n=45)$

\section{Boy}

Girl

$6(50 \%)$

$16(48.5 \%)$

$7(43.8 \%)$

$15(51.7 \%)$

\section{$0-5$ years}

6-12 years

$15(50 \%)$

$7(46.7 \%)$

Years since $\mathrm{SCl}$

$\begin{array}{lll}<1 \text { year } & 10(45.5 \%) & 12(54.5 \%) \\ 1-5 \text { years } & 12(52.2 \%) & 11(47.8 \%)\end{array}$

Level of injury $(n=45)$

$\begin{array}{lcr}\text { Paraplegia } & 21(52.5 \%) & 19(47.5 \%) \\ \text { Tetraplegia } & 1(20.0 \%) & 4(82.2 \%)\end{array}$

Completeness of injury $(n=45)$

Complete $\quad 14(48.3 \%) \quad 15(51.7 \%)$

Incomplete $\quad 8(50.0 \%) \quad 8(50.0 \%)$

$A / S(n=45)$

$\begin{array}{lcc}\text { A } & 14(48.3 \%) & 15(51.7 \%) \\ \text { B } & 0 & 1(100.0 \%) \\ \text { C } & 3(50.0 \%) & 3(50.0 \%) \\ \text { D } & 5(55.6 \%) & 4(44.4 \%)\end{array}$

Walking ability

$\begin{array}{llr}\text { Walkable } & 5(55.6 \%) & 4(44.4 \%)\end{array}$

All wheelchair
$9(56.3 \%)$

$14(48.3 \%)$

$15(50 \%)$

$8(53.3 \%)$

0.928

0.758

0.833

0.768

0.170

0.912

0.771

$19(52.8 \%)$
Abbreviations: AIS, American Spinal Injury Association Impairment Scale; SCI, spinal cord

injury; STAMP, Screening Tool for the Assessment of Malnutrition in Pediatrics.

aData are presented as the number of children with $\mathrm{SCl}$; percentage within nutritional

classification in parentheses.

${ }^{b} P$-values were determined with the use of Pearson chi-square test for all outcomes. category and walking ability). SCI of varying characteristics had the same probability of leading to a risk of undernutrition (Table 3 ).

\section{Nutritional status in Chinese children with SCI}

Regarding the nutritional status of pediatric SCI patients, 20 patients (44.4\%) were found to have a normal status, $12(26.7 \%)$ were found to have mild undernutrition, $3(6.7 \%)$ showed moderate undernutrition, $6(13.3 \%)$ were overweight and $4(8.9 \%)$ were obese (Figure 3). We also observed the trend of nutritional status after SCI and found that, as the duration of SCI increased, the WAZ/BAZ value increased and the HAZ value decreased. However, no significant correlations were identified between these parameters (Figure 4).

\section{Comparison of categorical variables on nutrition status and malnutrition risk}

According to calculated $z$-scores, $30(66.7 \%)$ children showed no malnutrition, $12(26.7 \%)$ children exhibited moderate malnutrition and $3(6.7 \%)$ children had severe malnutrition. According to STAMP scores, 22 (48.9\%) children ranked as low risk, 18 (40.0\%) as medium risk and $5(11.1 \%)$ as high risk (Figure 5). As an indicator of the overall criterion validity, 37 of the $45(82.2 \%)$ patients were classified as having the same degree of nutritional risk and undernutrition in both STAMP and $z$-score assessments. The agreement between the nutrition status and malnutrition risk was moderate $(k=0.603)$. The sensitivity and specificity of STAMP in the diagnosis of undernutrition were $100 \%$ and $73.3 \%$, respectively. The PPV of the same scores was $65.2 \%$, and the NPV was $100 \%$ (Table 4).

\section{DISCUSSION}

The data from the present study showed that subgroups of children with SCI had the same nutritional status and risk of malnutrition. The decreasing trends in nutritional status and poor appetite level following SCI require special attention. The STAMP may be a useful tool for identifying nutritional status in children with SCI.

Malnutrition, including both undernutrition and overnutrition, is associated with poor clinical outcomes, including increased mortality. In addition, malnutrition is associated with prolonged hospital stay, delayed recovery, increased care costs, ${ }^{19}$ poor somatic growth and development and reduced or delayed mental and psychomotor development. ${ }^{4}$ To prevent malnutrition, early identification of nutritional depletion is essential, ideally on admission to the hospital. Such an approach provides the physician with an opportunity to implement appropriate nutritional interventions, in the hope of preventing the worsening of undernourishment or decrease its severity when it is already present. ${ }^{9}$

The present study is the first study to assess the prevalence of malnutrition risk in hospitalized pediatric SCI patients in China. A high percentage of patients at risk for undernutrition (STAMP $\geqslant 2$, $51.1 \%$ ) was observed among the study population, which is similar to the observations in the UK's National Spinal Injuries Centre. ${ }^{5}$ There is a definite need to address the nutritional requirements of this special population group in China.

Our study also showed that preschool-age children and school-age children of different genders had similar WAZ, HAZ and BAZ values and STAMP scores. Usually, BMI/WAZ and HAZ are considered hallmarks of current and chronic nutrition status. ${ }^{10,20}$ A longstanding assumption is that by school age a child has survived the most critical period, and he/she is no longer vulnerable to undernutrition. This has led to the neglect of this specific group of children by researchers, health-care professionals and caregivers ${ }^{21}$ while assessing the nutrition status. Accordingly, school-age children with an SCI may have the 


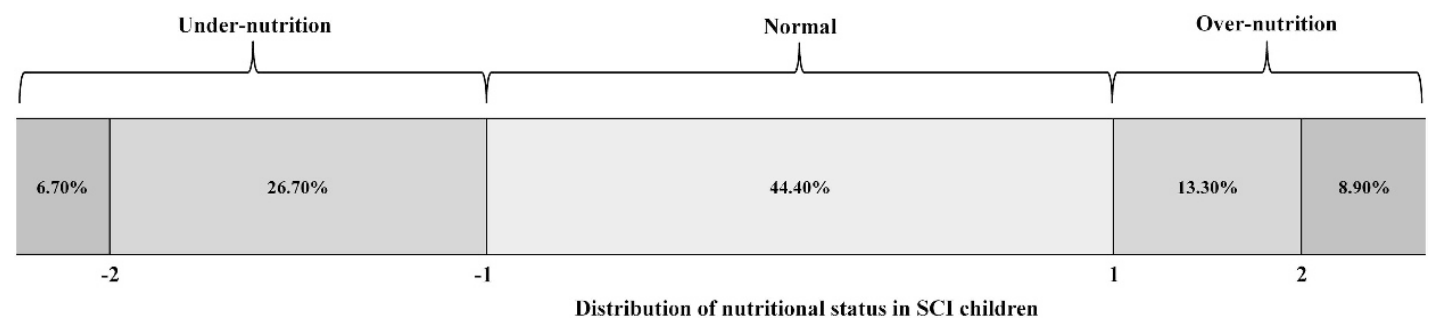

Figure 3 Distribution of nutritional status in $\mathrm{SCl}$ children.

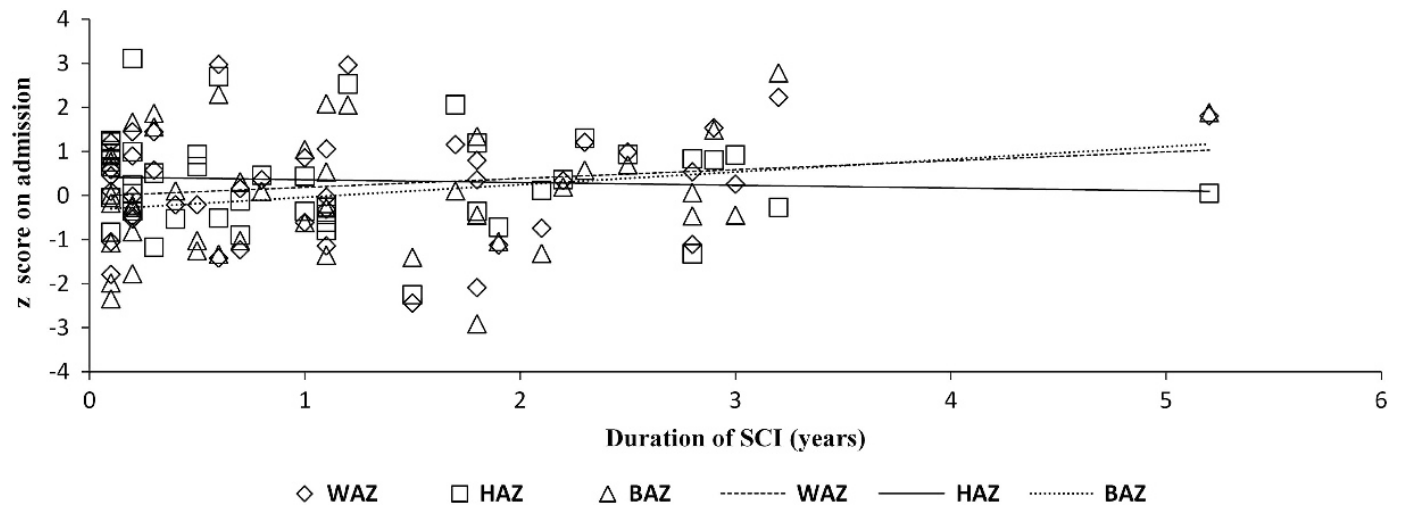

Figure 4 Correlation between duration of $\mathrm{SCl}$ and $z$-scores of WAZ, HAZ and BAZ at hospital admission.

better nutritional status (higher WAZ, HAZ and BAZ) and lower risk of malnutrition (lower STAMP) than preschool-age children, but this was not found in our study. All groups of children with SCI are vulnerable to malnutrition, ${ }^{22}$ and thus the nutritional status/risk in children of all age groups cannot be ignored.

Our results demonstrated that the degree of undernutrition risk was inversely proportional to the WAZ, HAZ and BAZ values and appetite level. As the classification of STAMP correlated with the degree of nutrition status, STAMP can be applied as an alternative method for assessment of nutrition status. In children with SCI, it is clear that risk of malnutrition on admission does necessarily predict a poor nutritional intake (low percentage of eating) during SCI. ${ }^{23}$ Low appetite level may represent the risk of malnutrition, which was confirmed in our study. Children have a high energy need per unit of body mass compared with adults and have limited energy reserves. Furthermore, children, unlike adults, need energy for growth, which makes them particularly vulnerable to malnutrition. ${ }^{24}$ For this reason, the likelihood of developing a risk for nutritional deficiencies increases when the child with a poor appetite is admitted to the hospital. This highlights the importance of assessing appetite during SCI and maintaining the adequate nutrition intake. Unfortunately, there are no accepted tools to identify the SCI patients with poor appetite now. ${ }^{23}$ Although the method of estimation may not be able to accurately reflect the actual appetite level, it may be a valid and simple alternative method at present.

The demographic/SCI characteristics of the patient population, such as age, gender, duration of SCI, motor scores, cause of injury, years since SCI, level of injury, AIS and walking ability, showed no correlations with nutritional risk. The groups of new injuries ( $\leqslant 1$ years) and old injuries ( $>1$ years), paraplegic and tetraplegic, walkable and wheelchair-bound patients showed the same risk of malnutrition. Hence, screening of malnutrition risk in all pediatric SCI patients seems to be mandatory even in children without severe injury,

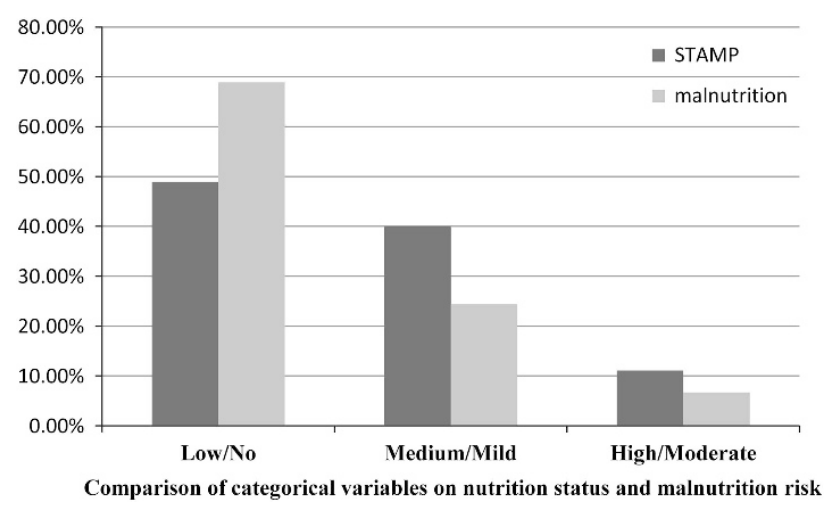

Figure 5 Comparison of categorical variables on nutrition status and malnutrition risk.

because the risk for malnutrition in the group with mild SCI might be overlooked. $^{25}$

Studies have also shown the coexistence of undernutrition and overnutrition, the latter manifesting as overweight and obesity among SCI patients, including children and adults. For pediatric SCI (the median duration of SCI was 4 years, with an interquartile range of $2.0-8.3$ years), undernutrition risk was $47.1 \%$ (STAMP $\geqslant 2$ ) and the prevalence of overnutrition was also high (overweight, 41.1; obese, $25.5 \%) .{ }^{4}$ Among adult SCI patients, $40-50 \%$ of patients are at risk for nutritional deficiency and $40-66 \%$ are at risk for obesity. ${ }^{26}$ The findings of the present study also reveal that both extremes of malnutrition exist among children with SCI. However, undernutrition is more prevalent than overnutrition. The prevalence of malnutrition (55.6\%), including undernutrition (33.4\%) and overnutrition $(22.2 \%)$, among pediatric SCI patients in this study is higher than that reported for hospitalized children in $\mathrm{China}^{27}$ and the prevalence of malnutrition (44\%) in adult patients with SCI. ${ }^{28}$ Hence, strategies 
Table 4 Applicability of the STAMP for assessment of nutritional status

\begin{tabular}{|c|c|c|c|c|c|c|}
\hline \multirow[t]{3}{*}{ Nutritional status } & \multicolumn{4}{|c|}{ Risk of nutrition (STAMP) } & \multirow[t]{3}{*}{ P-value } & \multirow[t]{3}{*}{ Kappa } \\
\hline & Low & Medium & High & Total & & \\
\hline & $(n=22)$ & $(n=18)$ & $(n=5)$ & $(\mathrm{N}=45)$ & & \\
\hline No undernutrition & $22(73.3 \%)$ & $8(26.7 \%)$ & 0 & 30 & 0.000 & 0.603 \\
\hline Mild undernutrition & 0 & $10(83.3 \%)$ & $2(16.7 \%)$ & 12 & & \\
\hline Total & 22 & 18 & 5 & 45 & & \\
\hline Agreement & $82.2 \%$ & & & & & \\
\hline Sensitivity & $100.0 \%$ & & & & & \\
\hline Specificity & $73.3 \%$ & & & & & \\
\hline Positive-predictive value & $65.2 \%$ & & & & & \\
\hline Negative-predictive value & $100 \%$ & & & & & \\
\hline
\end{tabular}

Abbreviation: STAMP, Screening Tool for the Assessment of Malnutrition in Pediatrics.

$P$-values were determined with the use of Fisher's exact test for all outcomes.

to improve the nutritional status (undernutrition and overnutrition) of pediatric SCI patients need to be strengthened.

The duration of an SCI may have negatively affected the nutritional status of the children enrolled in the study, inducing increases in the possibility of overweight (higher BAZ/WAZ) and stunting (lower HAZ). The impairment of the HAZ rate indicates that the growth of a child affected by longstanding diseases reflects deficient health and feeding conditions. The observation in the present study that HAZ decreases with increasing duration of SCI indicates that the existing undernutrition may be sustained or even deteriorate following SCI without nutritional therapy, resulting in chronic malnutrition or stunting. This deficiency in the WAZ/BAZ reflects a more recent impairment in nutritional state or wasting. ${ }^{29}$ On the contrary, the excess in WAZ /BAZ may indicate overweight or obesity.

The increase in the percentage of weight gain in relation to duration of SCI has been described in adults, ${ }^{2}$ but such an observation in a pediatric population has not been reported to date. A tendency for weight gain in pediatric SCI patients was observed in our study. Reduced food intake, reduced activity, loss of muscle mass and a decrease in energy requirements may be related to weight loss during the acute phase of SCI. ${ }^{4,25}$ However, in the long term, prolonged inactivity following an SCI can cause a shift in body composition and muscle atrophy, as well as excessive fat accumulation leading to weight gain. ${ }^{25,28}$ However, a lack of education and/or awareness of nutritional needs after SCI may all contribute to overweight and obesity. ${ }^{2}$ In short, the energy intake and nutritional requirements depend on the phase of the SCI. ${ }^{28}$

As mentioned above, the STAMP categories were associated with the degree of nutrition status. The STAMP tool showed moderate agreement with nutritional status assessed by WAZ/HAZ and BAZ ( $k$ : 0.603 , sensitivity: $100 \%$, specificity: $73.3 \%$ ), suggesting that it could be a useful tool with good sensitivity and specificity for identifying nutritional status. The higher sensitivity and PPV of STAMP reflect a greater likelihood that a child who is identified as being at risk of undernutrition using the tool will indeed be in an undernutrition state. This, coupled with the high specificity and NPV, reduces the risk of overidentifying undernutrition in these children.

The strength of the present study is that it has made a significant contribution in providing the much-needed data as evidence of the high double burden of malnutrition among children with SCI. Children with SCI are a heterogenous group, some of whom are malnourished and some of whom are overweight or obese. Strategies for improving the nutritional status of children with SCI cannot remain focused only on undernutrition; they also need to emphasize approaches for the prevention of overweight and obesity as well.

Our study has few limitations. The small sample size (excluding outpatients) from a single center may not be representative of the whole pediatric population with SCI in China. Further, to compare the relationship between nutritional status and SCI characteristics (such as duration of SCI) owing to a particular small data set is limited. The inhomogeneity of the group owing to inclusion of a few tetraplegic patients and the variability in the AIS classification might have influenced the outcome. The environment, social/psychological issues and eating habits could also influence the results. The estimation of the appetite level depended upon the proxy reporting by the parents or main caregivers. Few parents expressed difficulty in accurately estimating the appetite of their children. The dependence on third parties (caregivers or parents) to report food intake of the children may have led to a biased estimation of appetite to some extent, but the result that the risk of undernurtrition was associated with poor appetite concurs with the findings of other studies. ${ }^{30}$ This study did not include objective assessments of nutrition (such as biochemical measures or body density assessment) but was intended to evaluate the functionality and usefulness of the previously validated screening tools. Finally, the cross-sectional design of our study does not permit deriving causal relationships between malnutrition risk and undernutrition/poor outcome. ${ }^{12}$

Future research should determine the biochemical and/or clinical implications of nutrient inadequacies. In addition, research on inadequacies in relation to the different food groups, dietary patterns and eating behaviors, as well as the effects of supplements on nutritional status, is warranted. In conclusion, our findings reveal that screening of the nutritional status of all newly admitted pediatric SCI patients, including those of AIS D, is necessary, and somatic growth may decelerate over time after SCI. Finally, the STAMP, which is a tool for evaluating malnutrition risk, appeared to be a useful tool for assessing nutritional status in children with an SCI.

\section{DATA ARCHIVING}

There were no data to deposit. 


\section{CONFLICT OF INTEREST}

The authors declare no conflict of interest.

\section{ACKNOWLEDGEMENTS}

This study was supported by China Rehabilitation Research Center (2015CZ-7).

1 Wong S, Derry F, Graham A, Grimble G, Forbes A. An audit to assess awareness and knowledge of nutrition in a UK spinal cord injuries centre. Spinal Cord 2012; 50 446-451.

2 Sarni RO, Carvalho Mde F, Monte CM, Albuquerque ZP, Souza FI. Anthropometric evaluation, risk factors for malnutrition, and nutritional therapy for children in teaching hospitals in Brazil. J Pediatr (Rio J) 2009; 85: 223-228.

3 Keusch GT. The history of nutrition: malnutrition, infection and immunity. J Nutr 2003; 133: 336S-340S

4 Wong S, Graham A, Harini SP, Grimble G, Forbes A. Profile and prevalence of malnutrition in children with spinal cord injuries-assessment of the Screening Tool for Assessment of Malnutrition in Paediatrics (STAMP). Spinal Cord 2012; 50: 67-71.

5 Wong S, Graham A, Hirani SP, Grimble G, Forbes A. Validation of the Screening Tool for the Assessment of Malnutrition in Paediatrics (STAMP) in patients with spinal cord injuries (SCls). Spinal Cord 2013; 51: 424-429.

6 Sikorova L, Zavrelova B. Evaluation of malnutrition of hospitalized children by the Screening Tool for the Assessment of Malnutrition in Paediatrics. Cas Lek Cesk 2012; 151: 397-400.

7 Li JJ, Zhao L, Hong L, Li BR, Qian J, Ren $\mathrm{H}$ et al. Nutritional risk screening in critically ill children using Screening Tool for the Assessment of Malnutrition in Pediatrics. Chin J Clin Nutr 2014; 22: 323-328.

8 Chen FX, Lin ZH, Wu B. Influence factors of malnutrition risk in hospitalized children. Chin J Clin Nutr 2015; 23: 17-22.

9 Hulst JM, Zwart H, Hop WC, Joosten KF. Dutch national survey to test the STRONGkids nutritional risk screening tool in hospitalized children. Clin Nutr 2010; 29: 106-111.

10 Moeeni V, Walls T, Day AS. Assessment of nutritional status and nutritional risk in hospitalized Iranian children. Acta Paediatr 2012; 101: e446-e451.

11 Walters JL, Buchholz AC, Martin Ginis KA, Group S-SR. Evidence of dietary inadequacy in adults with chronic spinal cord injury. Spinal Cord 2009; 47: 318-322.

12 Marginean O, Pitea AM, Voidazan S, Marginean C. Prevalence and assessment of malnutrition risk among hospitalized children in Romania. J Health Popul Nutr 2014; 32: 97-102.

13 Secker DJ, Jeejeebhoy KN. Subjective Global Nutritional Assessment for children. Am J Clin Nutr 2007; 85: 1083-1089.

14 McCarthy H, Dixon M, Crabtree I, Eaton-Evans MJ, McNulty H. The development and evaluation of the Screening Tool for the Assessment of Malnutrition in Paediatrics (STAMP(c)) for use by healthcare staff. J Hum Nutr Diet 2012; 25: 311-318.
15 Poh BK, Ng BK, Siti Haslinda MD, Nik Shanita S, Wong JE, Budin SB et al. Nutritional status and dietary intakes of children aged 6 months to 12 years: findings of the Nutrition Survey of Malaysian Children (SEANUTS Malaysia). Br J Nutr 2013; 110 (Suppl 3): S21-S35.

$16 \mathrm{Li} \mathrm{H}$, Ji CY, Zong XN, Zhang YQ. Height and weight standardized growth charts for Chinese children and adolescents aged 0 to 18 years. Zhonghua Er Ke Za Zhi 2009; 47: 487-492.

17 Mushtaq MU, Gull S, Khurshid U, Shahid U, Shad MA, Siddiqui AM. Prevalence and socio-demographic correlates of stunting and thinness among Pakistani primary school children. BMC Public Health 2011; 11: 790.

18 Mushtaq MU, Gull S, Mushtaq K, Abdullah HM, Khurshid U, Shahid U et al. Height, weight and $\mathrm{BMI}$ percentiles and nutritional status relative to the international growth references among Pakistani school-aged children. BMC Pediatr 2012; 12: 31.

19 Hartman C, Shamir R, Hecht C, Koletzko B. Malnutrition screening tools for hospitalized children. Curr Opin Clin Nutr Metab Care 2012; 15: 303-309.

20 Spagnuolo MI, Liguoro I, Chiatto F, Mambretti D, Guarino A. Application of a score system to evaluate the risk of malnutrition in a multiple hospital setting. Ital J Pediatr 2013; 39: 81.

21 Ene-Obong H, Ibeanu V, Onuoha N, Ejekwu A. Prevalence of overweight, obesity, and thinness among urban school-aged children and adolescents in southern Nigeria. Food Nutr Bull 2012; 33: 242-250.

22 Wong S, Derry F, Jamous A, Hirani SP, Grimble G, Forbes A. The prevalence of malnutrition in spinal cord injuries patients: a UK multicentre study. Br J Nutr 2012; 108: 918-923.

23 Young AM, Kidston S, Banks MD, Mudge AM, Isenring EA. Malnutrition screening tools: comparison against two validated nutrition assessment methods in older medical inpatients. Nutrition 2013; 29: 101-106.

24 Joosten KF, Hulst JM. Prevalence of malnutrition in pediatric hospital patients. Curr Opin Pediatr 2008; 20: 590-596.

25 Campanozzi A, Russo M, Catucci A, Rutigliano I, Canestrino G, Giardino I et al. Hospital-acquired malnutrition in children with mild clinical conditions. Nutrition 2009; 25: 540-547.

26 Dionyssiotis Y. Malnutrition in spinal cord injury: more than nutritional deficiency. J Clin Med Res 2012; 4: 227-236.

27 Rasmussen $\mathrm{HH}$, Kondrup J, Staun M, Ladefoged K, Kristensen H, Wengler A. Prevalence of patients at nutritional risk in Danish hospitals. Clin Nutr 2004; 23: 1009-1015.

28 Wong S, Derry F, Grimble G, Forbes A. How do spinal cord injury centres manage malnutrition? A cross-sectional survey of 12 regional centres in the United Kingdom and Ireland. Spinal Cord 2012; 50: 132-135.

29 De Moraes Silveira CR, De Mello ED, Antonacci Carvalho PR. Evolution of nutritional status of pediatric in patients of a tertiary care general hospital in Brazil. Nutr Hosp 2008; 23: 599-606.

30 Lotfy HM, Sabry SM, Ghobrial EE, Abed SA. The effect of regular hemodialysis on the nutritional status of children with end-stage renal disease. Saudi J Kidney Dis Transp/ 2015; 26: 263-270. 